\title{
ONE FOR ALL- INTRODUCING AN ALTERNATIVE EASILY-ASSEMBLED ADJUSTABLE LOOP SUCTION DEVICE FOR DENTAL PROCEDURES
}

\author{
Tzong-Jeng Wu1, Szu-Yun Hsiao², Chung-Tzu Hsueh³, Yu-Pin Feng ${ }^{4}$
}

${ }_{1}^{1}$ Senior Consultant Anaesthesiologist, Department of Anaesthesiology, Cathay General Hospital, Taipei, Taiwan.

${ }^{2}$ Clinical Anaesthesiologist, Department of Anaesthesiology, Kinmen Hospital, Ministry of Health and Welfare, Taiwan.

${ }^{3}$ Consultant Dentist, Department of Dentistry, Cathay General Hospital, Taipei, Taiwan.

${ }^{4}$ Consultant Anaesthesiologist, Department of Anaesthesiology, Cathay General Hospital, Taipei, Taiwan.

HOW TO CITE THIS ARTICLE: Wu TJ, Hsiao SY, Hsueh CT, et al. One for all- introducing an alternative easily-assembled adjustable loop suction device for dental procedures. J. Evolution Med. Dent. Sci. 2018;7(30):3443-3444, DOI: 10.14260/jemds/2018/775

\section{BACKGROUND}

During dental sedation, control of the cough reflex is crucial for a safe and smooth procedure. Accumulated saliva is one of the predisposing factors for coughing. Therefore, extensive suction is very important. Using of standard saliva ejector may not be enough. We introduce hand-made Adjustable Loop Suction (ALS) as an alternative solution. The features of this device are easy assembly, single use, effective suction, excellent moldability, and customised smaller sizes for young children. The suction orifice near the posterior oral cavity effectively removes the fluid and the bent suction loop assists stability in the patient's mouth.1,2

In dental treatments, adequate removal of fluids accumulated in the oropharyngeal space is of critical importance. Failure to do so risks triggering the cough reflex, and predisposes choking of the patient, laryngospasm or oxygen desaturation. In these situations, the operator would be forced to remove the patient's mouth gag to allow the fluids to be expelled. Thus, an oral evacuation system which can effectively and efficiently drain fluids would not only greatly facilitate the procedure by providing clear visibility and lowering the frequency of interruptions, but more importantly improves patient safety by reducing stimulation of the cough reflex.

Here, we introduce a hand-made Adjustable Loop Suction Apparatus (ALSA) (Fig. 1 A-C) as an alternative solution to the conventional suctioning devices. It is easily assembled from materials accessible to medical providers. A common PAHSCO FR 14 (green) cap-cone suction catheter is used. Seven pairs of holes $(1.0 \times 1.0 \mathrm{~mm})$ are perforated along the anterior portion of the suction catheter at $1.0 \mathrm{~cm}$ distance apart. A pliable surgical stainless-steel wire (20 gauge) is inserted as a stylet for shaping the curve of the loop. To complete the loop, the end of the suction catheter is inserted into the hole on its plastic cap-cone connector. Finally, the anterior part of the loopedsuction is bent to create a spacer, which can anchor to the opposite row of teeth. ${ }^{3}$ The shape and curve of this ALSA can be easily adjusted as needed during the procedure.

'Financial or Other Competing Interest': None.

Submission 15-06-2018, Peer Review 09-07-2018,

Acceptance 15-07-2018, Published 23-07-2018.

Corresponding Author:

Yu-Pin Feng,

Consultant Anaesthesiologist,

Cathay General Hospital,

No.280, Sec. 4, Ren'ai Road,

Da'an District, Taipei City

Taiwan.

E-mail:fengyupin0507@gmail.com

DOI: $10.14260 /$ jemds $/ 2018 / 775$
Existing saliva ejectors cannot effectively remove the cumulated fluids at the posterior oral cavity. Commercial products such as Mr. Thirsty prevent fluids and debris from being aspirated or swallowed, but does not fit to every patient's mouth resulting in inadequate vacuuming of retropharyngeal fluid. Others like Fastdam TM cannot be stably anchored. Coolex TM saliva ejector offers effective removal of secretions with flexibility and shape retainability, but comes at a high cost (\$274.95 329.5).4,5 Another problem is that Coolex TM was designed to be reused, but may not always be easily disassembled for disinfecting, which raises concerns of possible cross-contamination between patients. Furthermore, its silicone material may deteriorate with repeated exposure in the autoclave and cracks may form at the suction holes with multiple use (Fig. 1D).

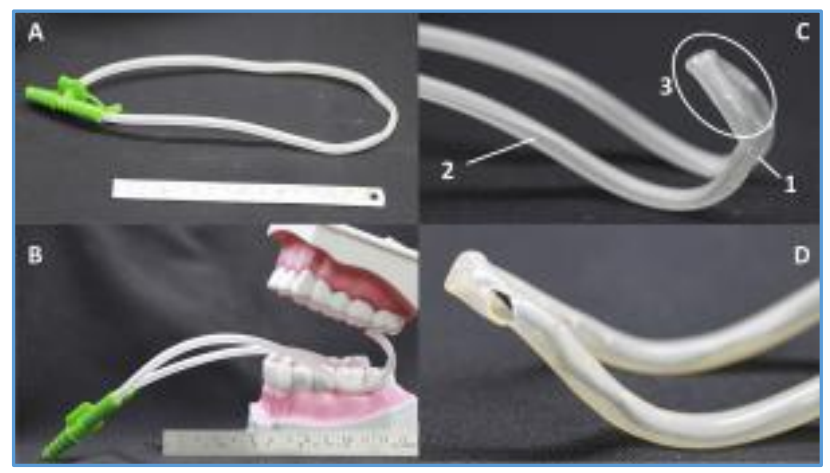

Figure 1. Adjustable Loop Suction Apparatus (ALSA; A-C) and commercial Silicone Suction Device (D). ALSA comprises of a looped hollow tube made with a cap-cone type suction catheter along with several suction holes. The tube is embedded with a Surgical Wire (20 gauge), which has both Flexibility and Moldability. The side holes (1.0 $\mathrm{x}$ $1.0 \mathrm{~mm}$ ) in the anterior part of the device need to be small enough to maintain sufficient continuous negative pressure for suctioning (C-1). 20-gauge medical-use Sstainless steel wire as a stylet to shape the curve of the loop (C-2). The Spacer acts as an anchor to provide fixation during the Procedure (C-3). Silicone Material deteriorates with repeated exposure in the autoclave, and cracks may form at the Suction Holes (D).
Features of ALSA
1. Easily assembled in the operating room.
2. Single use with no cross-contamination.
3. Spacer can be securely anchored onto the opposite teeth.
4. Effective suction, especially for posterior oral cavity procedures.
5. Flexibility and excellent moldability.
6. Customised smaller sizes for toddlers. 
Our ALSA offers dentists an effective way to drain oral cavity liquids during treatment. The suction orifice near the posterior oral cavity effectively removes the fluid and the bent suction loop assists with stability in the patient's mouth. The device is easy to place, and the operator can shape the suction apparatus according to each patient's needs. It readily connects to conventional suction systems.

\section{REFERENCES}

[1] Kohjitani A, Egusa M, Shimada M, et al. Accumulated oropharyngeal water increases coughing during dental treatment with intravenous sedation. J Oral Rehabil 2008;35(3):203-8.

[2] Hanamoto H, Sugimura M, Morimoto Y, et al. Cough reflex under intravenous sedation during dental implant surgery is more frequent during procedures in the maxillary anterior region. J Oral Maxillofac Surg 2013;71(4):e158-63.
[3] APT. Inc Multi-Purpose Vacuum Instrument Coolex. Accessed on December 27, 2017. http://www.apt-inc.jp/zoo/emulti/

[4] American Dental Accessories Coolex Saliva Ejector. Accessed on December 27, 2017. https://www.amerdental.com/suctionsystems/coolex-saliva-ejector-0-75-connector.html

[5] Sasaki Y, Kato S, Miura M, et al. Correlation between body movements and salivary secretion during sedation. Anesth Prog 2016;63(4):185-91. 